\title{
603 TARGETED STAT3 DEGRADATION LEADS TO REMODELING OF AN IMMUNOSUPPRESSIVE TUMOR MICROENVIRONMENT AND SUBSEQUENT SENSITIZATION TO IMMUNE CHECKPOINT THERAPY
}

Joyoti Dey*, Phillip Liu, Michele Mayo, Rahul Karnik, Bin Yang, Vaishali Dixit, Jieun Jeong, Jared Gollob, Chris De Savi. Kymera Therapeutics, Watertown, MA, USA

Background Signal Transducer and Activator of Transcription 3 (STAT3), a multifaceted transcription factor, is aberrantly activated across a variety of malignancies; however, its selective targeting has to-date remained a therapeutic challenge. STAT3 plays a pivotal role in shaping the tumor immune landscape through cancer cell-intrinsic mechanisms, direct regulation of immune cell function and via cancer cell- tumor microenvironment (TME) crosstalk, that collectively result in an immunosuppressive TME. Targeted protein degradation represents a novel therapeutic modality enabling direct targeting of previously undruggable oncoproteins. We have developed potent and selective STAT3 heterobifunctional degraders demonstrating activity across diverse tumor and immune cell types.

Methods We investigated the immunomodulatory impact of STAT3 degradation on tumorigenesis in syngeneic mouse models representing cancers with heterogeneous immune milieus. Methods included in vivo pharmacological approaches, immunophenotyping and gene expression profiling.

Results Treatment of CT-26 (colorectal cancer) and A20 (B-cell lymphoma) tumor-bearing mice with a STAT3 degrader resulted in significant tumor growth inhibition compared to controls, with loss of STAT3 protein in both tumor cells and TME. This was accompanied by a decrease in M2 polarized macrophages and concomitant increases in M1 polarized macrophages and tumor infiltrating lymphocytes. The anti-tumor responses were abrogated by antibody mediated CD8 $+\mathrm{T}$ cell depletion or by using immunodeficient host-strains implicating the observed efficacy to be predominantly driven by immunedirected mechanisms. Gene expression profiling of STAT3 degrader-treated CT-26 tumors showed marked increases in proinflammatory genes including $\mathrm{T}$ cell and M1 macrophage activation markers, compared to controls. Notably, induction of an Ifn $\gamma$-responsive gene signature (Ifn $\gamma$, Stat1, Cxcl9, Cxcl10, Ido1) suggested that STAT3 degradation results in a T-cell inflamed phenotype associated with responsiveness to immune checkpoint therapy (ICT). Furthermore, on-treatment tumors showed an upregulation of genes such as Pdl1, Ctla4, Lag3 which reflect $\mathrm{T}$ cell activation as well as counterregulatory mechanisms. Therefore, we evaluated STAT3 degradation in combination with anti-PD1 in these models which are poorly responsive to anti-PD1 monotherapy. Robust synergy was observed in the CT-26 model with $60 \%$ complete responses and development of immunological memory as confirmed by tumor re-challenge studies. Studies are underway to ascertain the applicability of this combination therapy in different tumor-immune contextures and indications, and to elucidate the mechanistic basis of synergy.

Conclusions STAT3 degradation remodels an immunosuppressed TME activating anti-tumor immunity as monotherapy and effectively combines with anti-PD1. These data provide a rationale for selectively degrading STAT3 as a strategy to sensitize cancers with relevant immune contextures to ICT in the clinic.

http://dx.doi.org/10.1136/jitc-2021-SITC2021.603 